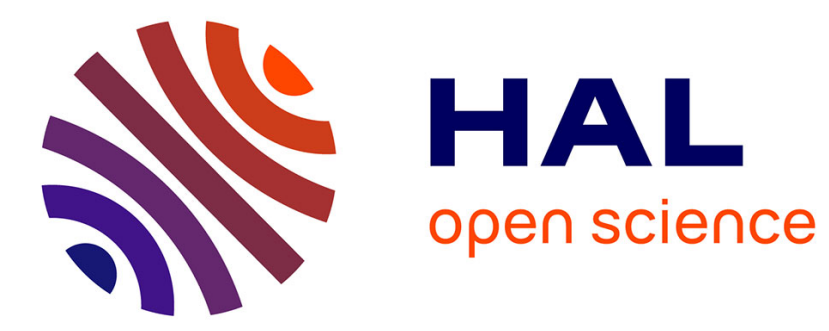

\title{
Charge state breeding with an ECRIS for ISAC at TRIUMF
}

\author{
F. Ames, K. Jayamanna, D.H.L. Yuan, M. Olivo, R. Baartman, P. Bricault, \\ M. Mcdonald, P. Schmor, T. Lamy
}

\section{- To cite this version:}

F. Ames, K. Jayamanna, D.H.L. Yuan, M. Olivo, R. Baartman, et al.. Charge state breeding with an ECRIS for ISAC at TRIUMF. 16. International Workshop on ECR Ion Sources, ECRIS '04, Sep 2004, Berkeley, California, United States. pp.147-150, 10.1063/1.1893385 . in2p3-00023381

\section{HAL Id: in2p3-00023381 https://hal.in2p3.fr/in2p3-00023381}

Submitted on 3 Dec 2004

HAL is a multi-disciplinary open access archive for the deposit and dissemination of scientific research documents, whether they are published or not. The documents may come from teaching and research institutions in France or abroad, or from public or private research centers.
L'archive ouverte pluridisciplinaire HAL, est destinée au dépôt et à la diffusion de documents scientifiques de niveau recherche, publiés ou non, émanant des établissements d'enseignement et de recherche français ou étrangers, des laboratoires publics ou privés. 


\title{
Charge State Breeding with an ECRIS for ISAC at TRIUMF
}

\author{
F. Ames, K. Jayamanna, D.H.L. Yuan, M. Olivo, R. Baartman, P. Bricault, M. \\ McDonald, P. Schmor
}

TRIUMF, 4004 Wesbrook Mall, Vancouver BC V6T 2A3, Canada

T. Lamy

LPSC UJF-IN2P3-CNRS, 53 Av. Des Martyrs, 38026 Grenoble, France

\begin{abstract}
For the acceleration of radioactive ions the usable mass range is limited by the A/q acceptance of the first accelerator stage. Since an efficient primary ion source normally produces singly charged ions, charge state breeding is necessary if higher masses are to be accelerated. At TRIUMF an ECR source has been chosen as a breeder due to its potential high efficiency in producing intermediate $\mathrm{A} / \mathrm{q}$ values. To minimize the necessity for further stripping an A/q around 6 is desirable. A $14 \mathrm{GHz}$ "PHOENIX" booster from Pantechnik has been set up on a test bench. The singly charged ions are produced from different ion sources, which can be mounted in a standard ISAC target-ion-source set-up. For the first tests an ECR source to produce noble gas beams has been chosen. The aim of the measurements at the test bench is to find the optimum operation conditions of the charge state booster and the injection and extraction ion optics. Working with radioactive ions always means that the system should aim for high efficiency, as the production of such species is limited. Therefore, special emphasis has to be put on the highest yield for the production of the desired charge state. A second point is the analysis of the extracted beam quality in order to optimize mass separation and transport efficiency. The paper shows the status of the set-up and reports on first results of the charge breeding of Ar, Ne and Xe. With Xe a total efficiency of $22.5 \%$ has been achieved.
\end{abstract}

\section{INTRODUCTION}

With the development of radioactive beam facilities at different laboratories charge state breading has become an important issue especially in connection with post acceleration. At an ISOL facility the ions are produced typically in a $1+$ state at an energy of several $10 \mathrm{keV}$. But in order to allow the acceleration to higher energies an increase in charge state is essential. There are several methods being used so far. The first one involves the stripping of the singly charged ions after an initial acceleration to some 100 $\mathrm{keV} / \mathrm{u}$. This method is limited to low masses, where the first acceleration is possible. At higher masses the only way is to increase the charge state before acceleration. In order to do so they are injected into an ion source for highly charged ions. There are two types of ion sources being used so far. An electron beam ion source EBIS has been used at the post accelerator REX-ISOLDE at CERN [1]. It can deliver beams of highly charged ions up to an efficiency of about $10 \%$ for a single charge state. The disadvantage of such a system is that it requires a pulsed beam at a small emittance for the incoming ions. That means a cooling and prebunching of the beam is necessary, which limits the total efficiency and the intensity to be used. If the source should run in a continuous mode the only solution so far is the use of an ECR [2], [3], [4]. The efficiency for the production of intermediate charge states has been reported as high as about $10 \%$ especially if noble gas ions are used and the system 
can be run also at high intensity. A disadvantage is the sometimes high background due to the ionization of the support gas or residual gas. Therefore a small emittance of the extracted beam is essential. This will reduce the background due to neighboring high intensity $\mathrm{A} / \mathrm{q}$ values or scattered ions in the beam lines.

\section{THE CHARGE STATE BOOSTER AT THE TRIUMF ION SOURCE TEST STAND}

At TRIUMF radioactive ions are produced at the ISAC facility by means of a high intensity $500 \mathrm{MeV}$ proton beam hitting a solid target. To produce singly charged ions a surface ion source and an ECR have been used so far. The maximum A/q ratio of 30 for the post acceleration is given by the acceptance of the first linear RFQ accelerator. To increase this number it is planned to introduce charge state breeding (CSB) with an ECR source. Therefore a $14 \mathrm{GHz}$ "PHOENIX" booster from Pantechnik has been set up first on a test bench. It can be equipped with several ISAC type ion sources. The 1+ section has already been described in detail in [5]. Some space in front of the CSB has been reserved to allow for the insertion of a beam cooling device. Following the CSB a double focusing mass spectrometer consisting of a $90^{\circ}$ magnetic and two $45^{\circ}$ electrostatic benders has been installed. Two electrostatic quadrupole doublets in front of the magnet ensure the focusing of the beam from the CSB to the object point of the spectrometer. The emittance of the incoming and outgoing beam can be measured at several points by means of inserting emittance meters [6]. The entire set-up is shown in figure 1 .

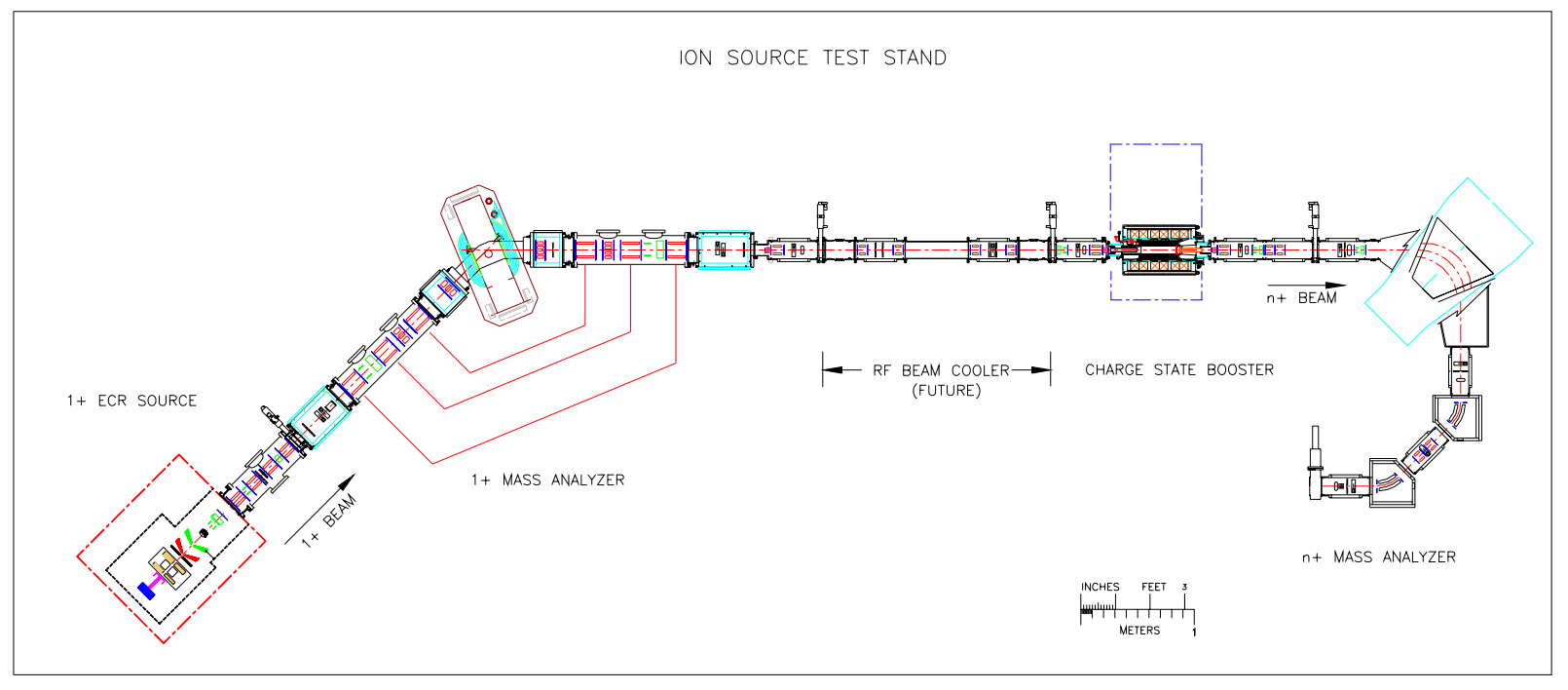

FIGURE 1. Set-up of the TRIUMF ion source test bench.

\section{Set-up of the n+ ECR source}

To meet the velocity acceptance of the ISAC RFQ accelerator with a mass to charge ratio around 7.5 the source has to be run at a potential of about $15 \mathrm{kV}$. The set-up of the PHOENIX ion source has been slightly changed for the requirements at TRIUMF. In order to limit the total emitted current the extraction aperture in the plasma electrode has been reduced from originally 
$8 \mathrm{~mm}$ diameter to $3.5 \mathrm{~mm}$. The extraction is done with one electrode on ground potential $12 \mathrm{~mm}$ in front of the extraction aperture. In order to protect the window, which insulates the source vacuum from the waveguide, from being damaged by the plasma, it has been placed outside the stray magnetic field of the source. Additionally, water cooling of the waveguide has been installed and the portion of the waveguide at high vacuum has been equipped with small holes and connected through a high voltage insulator to the vacuum chamber in front of the source for additional pumping. This guarantees a high vacuum inside the waveguide and thus minimizes the risk of a plasma discharge in it, which could destroy the window. The support gas is fed in directly into the plasma chamber at the injection side. On each side of the source pumping with both a $500 \mathrm{l} / \mathrm{s}$ turbo pump and a $2000 \mathrm{l} / \mathrm{s}$ cryo pump ensures a pressure below some $10^{-7}$ Torr.

\section{MEASUREMENTS AND RESULTS}

In first commissioning measurements the CSB has been run without injecting ions in order to optimize the extraction and analyzing ion optics. Ar or He has been used as a support gas. The efficiency and charge state distribution for the ionization of $\mathrm{Ar}$ for gaseous injection has been determined. The charge state distribution when using $\mathrm{He}$ as support gas and injecting Ar via a calibrated leak is shown in figure 2. The maximum in the distribution is at $7+$ with an efficiency of $2.7 \%$. Summing up the efficiency for all charge states gives a total efficiency of $12 \%$. For this measurement a microwave power of $100 \mathrm{~W}$ and a He flow of about $10^{-3}$ sccm have been used. This efficiency value includes the transmission of the extraction and analyzing system. Especially in the line in front of the magnet, the high beam current causes losses due to space charge effects. As the vacuum in the analyzer for these first measurements is only about $1 \cdot 10^{-6}$ Torr, charge exchange for the higher charge states will occur, resulting in an estimated loss of up to about $20 \%$.

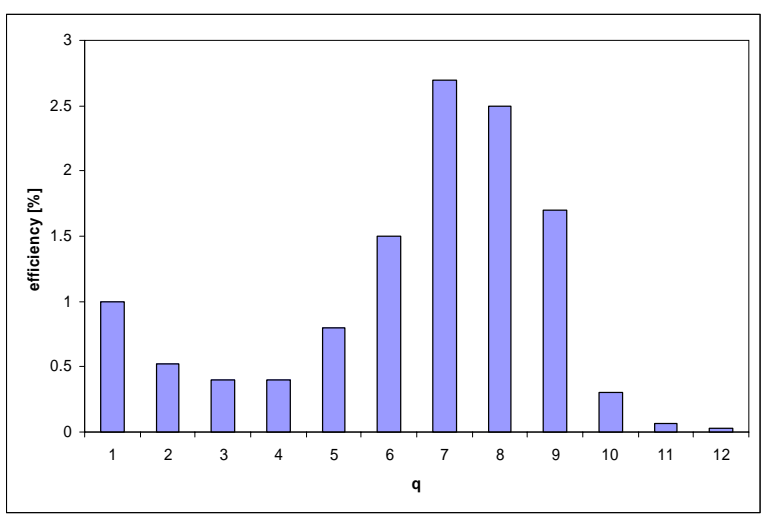

FIGURE 2. Charge state distribution of Ar when injected as a gas via a calibrated leak.

The emittances for the different charge states of Ar have been determined at the end of the analyzing system. Values up to $20 \pi \mathrm{mm}$ mrad at $15 \mathrm{kV}$ source potential have been found for $86 \%$ of the beam enclosed. Within the measurement accuracy of $\pm 10 \%$ no significant dependence on the charge state could be found.

For the charge breeding measurements an ECR [6],[7] source has been used to produce beams of singly charged $\mathrm{Ne}, \mathrm{Ar}$ and Xe ions. The CSB has been run with a low flow of He gas or with no additional support gas. First the potential of the CSB relative to the 1+ source has been changed to find the optimum deceleration parameter. An example of such a measurement is shown in figure 3. As has been reported from other groups, a broad structure can be found for noble gases with some saturation behavior at low potentials. This indicates the possibility for noble gases of being reemitted from the plasma chamber walls if their initial energy is too high for being captured by the plasma. 


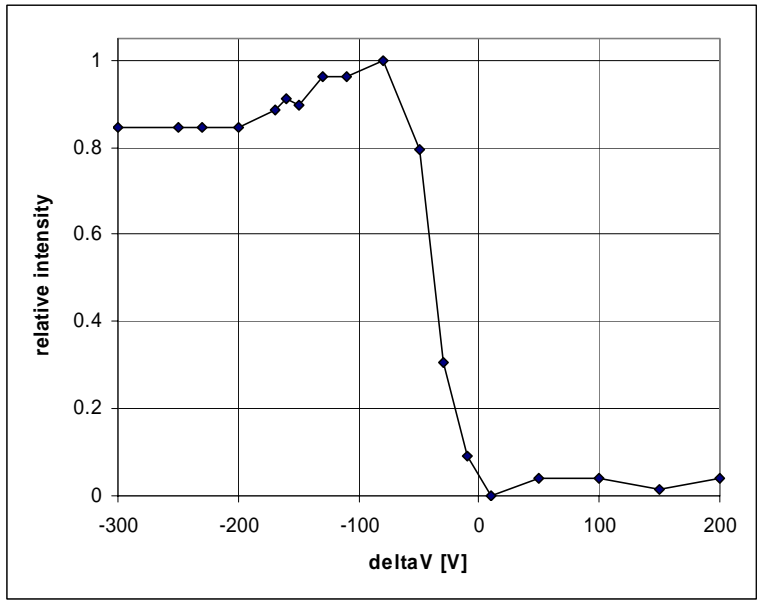

FIGURE 3. Relative intensity of the ${ }^{22} \mathrm{Ne}^{4+}$ signal as function of the CSB potential with respect to the $1+$ ion source.

After this the efficiencies for the different charge states have been determined. An example is shown in figure 4 for Xe. In this case the maximum was at 13+ with $3.7 \%$. Summing up all the charge states a total efficiency of $22.5 \%$ can be found. This measurement has been done with a microwave power of $250 \mathrm{~W}$ and no support gas. In the case of $\mathrm{Ar}$ and $\mathrm{Ne}$ the maximum in the distribution was at 8+ and 4+ respectively, with a smaller total efficiency, but at these measurements a microwave power of only $100 \mathrm{~W}$ could be used.

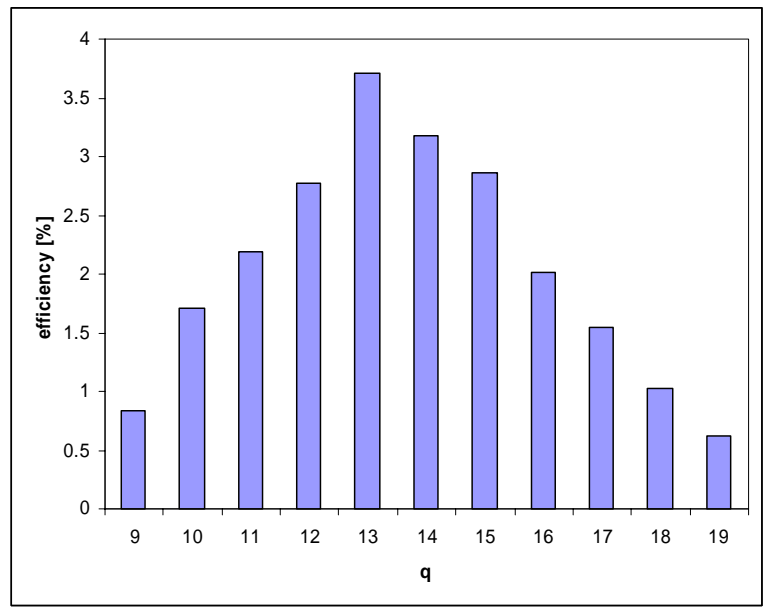

FIGURE 4. Efficiency of the charge state breeding of Xe for different charge states.

\section{CONCLUSION AND OUTLOOK}

The ECR charge state booster has been put successfully into operation at a test beam facility at TRIUMF. The first measurements could already demonstrate its capability of producing ions of intermediate charge states from singly ionized injected beams. The total efficiency is $22.5 \%$ for Xe and we expect this to improve. More studies on its dependence on the support gas or the composition on the residual gas in the plasma chamber will be done. Additionally the influence of the reduced extraction hole in the plasma electrode compared to the original design has to be studied in more detail with simulations and experimentally. Here a compromise between high extraction efficiency and a small emittance, which guarantees high transmission through the rest of the set-up, has to be found. In order to extend the measurements to non noble gas ions a surface ion source will be installed in the $1+$ line. In principle the efficiency can be increased by reducing the emittance of the incoming beam. This will be studied with the addition of a gas filled RFQ cooler [9] in front of the CSB. Such a device is being presently constructed at TRIUMF. Especially the overall efficiency with this system and the reliability for an on-line operation together with the CSB has to be studied.

\section{ACKNOWLEDGMENTS}

TRIUMF receives federal funding via a contribution agreement through the national research Council of Canada.

\section{REFERENCES}

1. Ames F. et al., Rev. Sci. Instr. 75 1607-1609 (2004)

2. Sortais P. et al., Nucl. Phys. A 701 537-549 (2002)

3. Lamy T. et al., Rev. Sci. Instr. 75 1624-1626 (2004)

4. Jeong S.C. et al., Rev. Sci. Instr. 75 1631-1633 (2004)

5. Dombsky M. et al., Nucl. Instr. Meth. B 126 50-54 (1997)

6. Yuan D. et al. Rev. Sci. Instr. 67 1275-1276 (1996)Bricault P., these proceedings

7. Jayamanna K. et al, Rev. Sci. Instr. 75 1621-1623 (2004)

8. Bricault P., these proceedings

9. Herfurth F., Nucl. Instr. Meth. B 204 587-591 (2003) 